\title{
Relationships between cognitive function and frontal grey matter volumes and thickness in middle aged and early old-aged adults: The PATH Through Life Study
}

\author{
Prapti Gautam ${ }^{\mathrm{a}, *}$, Nicolas Cherbuin ${ }^{\mathrm{a}}$, Perminder S. Sachdev ${ }^{\mathrm{b}, \mathrm{c}}$, Wei Wen ${ }^{\mathrm{b}, \mathrm{c}}$, Kaarin J. Anstey ${ }^{\mathrm{a}}$ \\ a Centre for Mental Health Research, Australian National University, Canberra, ACT, Australia \\ b School of Psychiatry, University of New South Wales, Sydney, Australia

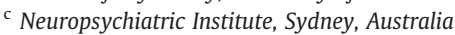

\section{A R T I C L E I N F O}

\section{Article history:}

Received 11 October 2010

Revised 21 December 2010

Accepted 9 January 2011

Available online $\mathrm{xxxx}$

\section{Keywords:}

Frontal cortex

Cognitive aging

MRI

\begin{abstract}
A B S T R A C T
The study examined the relationship of lateral frontal cortical volume and thickness with cognitive function in two samples of healthy middle aged (MA, 44-48 years old) and early old-age (OA, 64-68 years old) adults. T1-weighted magnetic resonance imaging scans were acquired in $400 \mathrm{MA}$ and $397 \mathrm{OA}$ adults from respective random community samples. Cortical volumes and thickness were measured with a surface-based segmentation procedure (http://surfer.nmr.mgh.harvard.edu). Volumes of lateral frontal grey matter were found to be significantly lower for OA than MA. Structure-function relationships were investigated using path analyses. In OA, smaller lateral frontal volumes were associated with better episodic memory $(E M)(p<0.012$, $\mathrm{B}=-0.117)$, and Symbol-Digit Modalities Test (SDM) $(\mathrm{p}<0.031, \mathrm{~B}=-0.118)$ performance. Smaller frontal cortical thickness was also associated with better EM $(\mathrm{p}<0.01)$ and SDM $(\mathrm{p}<0.01)$ performance in OA. However, in MA greater cortical thickness was associated with better EM and $(\mathrm{p}<0.01)$ and reaction time (RT) $(\mathrm{p}<0.01)$. OA cohort showed significant positive correlations between Total Brain Volume and SDM, DigitBackwards span and RT. Possible explanations and implications of the relationships in the context of cognitive aging in healthy adults, and limitations of cross-sectional research are discussed.
\end{abstract}

(c) 2011 Elsevier Inc. All rights reserved.

\section{Introduction}

The human brain undergoes structural and functional changes throughout the lifespan. In vivo (Courchesne et al., 2000; Ge et al., 2002) and post mortem (Riddle et al., 2010) studies of brain volumes have shown that grey matter starts declining slowly early on in life. White matter volume increases until adulthood and remains relatively stable until later adulthood where significant volume decline is apparent (Ge et al., 2002; Gogtay et al., 2004). The substrates for volume decline during normal aging consist of a combination of factors including reductions in myelin fibers (Marner et al., 2003), neuronal shrinkages and reduction of dendritic arborization and synaptic densities (Haug and Eggers, 1991) rather than significant neuronal loss (Freeman et al., 2008). The prefrontal and frontal lobes show increased age-related decline in comparison with other brain regions (Resnick et al., 2003) with age while cognitive aging is associated with declines in frontally mediated functions such as attention, response inhibition, processing speeds and episodic and working memory (Salthouse, 2003; West, 1996). In contrast to normal aging, brain atrophy rates are more rapid during pathological conditions such as Alzheimer's Disease (AD), and Mild

\footnotetext{
* Corresponding author at: Centre for Mental Health Research, Building 63, Australian National University, Canberra, ACT 0200, Australia. Fax: +61261250733. E-mail address: prapti.gautam@anu.edu.au (P. Gautam).
}

Cognitive Impairment (MCI) (Fox et al., 2000) with more severe memory symptoms that start primarily in the hippocampus and the medial temporal lobe but also involve other regions of the brain (Buckner, 2004; Davatzikos et al., 2008).

\section{Age, cognitive status and structure-function relationships}

The relationships between brain structure and cognitive performance have been found to differ depending on the cognitive status of the participants studied. In $\mathrm{AD}$ and $\mathrm{MCI}$ patients, atrophy of the hippocampus (as well as entorhinal and medial temporal cortices) have been found to associate strongly with cognitive decline (Rossi et al., 2007; Van Der Flier et al., 2002) with smaller volumes being associated with worse performance. In contrast, a meta-analyses investigating associations between hippocampal volume and memory measures in healthy participants, i.e. with no clinically significant neuropathology, only found weak associations when samples of young and old adults were considered, and a positive correlation when only older adults were considered (Van Petten, 2004). Similarly, structure-function relationships in young adults frequently report negative correlations, for instance, between memory and hippocampal as well as temporal lobe volumes (Chantôme et al., 1999; Foster et al., 1999; Sowell et al., 2001).

Regarding structure-function relationships of frontal volumes in healthy older adults, associations reported have been mixed. Various 
studies have found that larger lateral prefrontal cortex volumes correlate with lower perseverative errors (Gunning-Dixon and Raz, 2003; Head et al., 2009), as well as with better performance in visuospatial skills, working memory (Raz et al., 1999, 1998; Soderlund et al., 2004), episodic memory performance (Head et al., 2008) and fluid intelligence (Raz et al., 2008) while frontal lesions have been found to reduce performances in response inhibition and goal-setting (Eslinger et al., 2004; Picton et al., 2007). However, null relationships between working memory and prefrontal volumes (Raz et al., 1999; Sanfilipo et al., 2002) as well as negative associations have also been reported between regions of the prefrontal cortices and various cognitive measures in older adults (Duarte et al., 2006; ElderkinThompson et al., 2008; Salat et al., 2002; Van Petten et al., 2004). Van Petten et al. (2004) have stated similar observations concluding that while older adults with neuropathology generally display positive correlations, normal older adults seem to display mixed correlations and young adults frequently display negative correlations between frontal cortex structure and function.

\section{Rationalizations for structure-function relationships}

Various explanations have been proposed for the differences in direction of relationships seen in structure-function studies and have focused mainly on cognitive functions subserved by the temporal (including hippocampal) and frontal regions. The positive structurefunction relationships in the presence of neuropathology has been explained in terms of the 'bigger is better' hypothesis, i.e., larger hippocampal and cortical volumes are thought to equate to larger remaining portions of functional neural substrates and hence lead to better functionality. However, in normal aging the smaller volumetric differences observed might have non-pathological developmental origins which may have little or no functional effects: this would account for null structure-function associations in healthy older populations.

Negative structure-function associations in young subjects have been explained in terms of inadequate pruning (Chantôme et al., 1999; Foster et al., 1999). Since declines in grey matter volume in childhood are achieved through neural pruning, remnant larger regions during this period might be due to insufficient pruning causing poorer neural communication. In this context, it has been proposed that in healthy older adults structure-function relationships could be a result of poor pruning during development (Duarte et al., 2006) and/or other factors leading to pre-existing differences rather than age-associated cognitive decline (Shenkin et al., 2003; Staff et al., 2006; Van Petten et al., 2004), and hence negative relationships could still be observed in later life.

It is also possible that negative relationships could have been observed due to the effect/s of suppressor variables whose presence could significantly improve the associations between two variables (MacKinnon et al., 2000). For example, if a covariate also acts as a suppressor in the relationship such that irrelevant variance of the predicting cortical structure is suppressed, negative relationships could be seen during structure-function analyses. Lastly, the role of 'neural efficiency' has been described in healthy older adults with preserved cognitive functioning, where high cognitive reserves are associated with negative correlations between brain activation and cognitive performance (Bartrés-Faz et al., 2009; Solé-Padullés et al., 2009; Stern et al., 2005). Thus efficiency of cortical structures in cognitive functioning could be related not just to their size but their effectiveness.

\section{Age and frontal structure-function relationship in aging}

With age-related volume decline which is heterogeneous over the cortex, (Good et al., 2001; Raz et al., 2005), the neuromodulatory role of the frontal function on posterior regions could also be affected. The inhibitory influences of frontal regions which may be counterbalanced by activation in other cerebral regions in younger age groups could be responsible for inverse relationships in structurefunction studies in older adults if posterior regions experience atrophy and/or decrease in function. For instance, during memory retrieval inhibitory action of the frontal cortices on parietal regions has been shown to lead to the suppression of interfering information leading to better recall while increased inhibition from the dorsal and ventral prefrontal cortices can also cause forgetting leading to disruption in retrieval (Crescentini et al., 2010; Wimber et al., 2008). Thus feedback loops between the frontal cortices and other cerebral structures could be disturbed and lead to poorer cognitive outcomes not only in the case of frontal atrophy and decreased input to posterior regions but also in the case of an excessive, preserved inhibitory signal from the frontal cortices to more severely atrophied, less functionally active posterior regions. Successful completion of tasks such involving processes such as working memory requires the co-ordination of various structures, including the prefrontal and parietal regions and disturbance in either of these systems can lead to poorer performance (Müller and Knight, 2006; Soderlund et al., 2004).

Methodological explanations for discrepancies in structure-function relationships

Some of the discrepancy in structure-function relationships in older adults could have been brought about due to study designs/ methodological limitations. For example, in some studies (MacLullich et al., 2002; Schretlen et al., 2000), while frontal lobe volumes correlated positively with cognitive measures, such relationships were later found to be non-significant when Intra-Cranial Volume (ICV) was considered. Similarly, while other studies reporting positive correlations normalized for body size (Gunning-Dixon and Raz, 2003; Head et al., 2009), they used samples with broad age-ranges. In such samples, spurious correlations can occur with unrelated variables due to effects of age (Hofer and Sliwinski, 2001) leading to discrepant results in structure-function relationships, especially in cross-sectional studies as individual differences cannot all be controlled for.

Additionally, because regions of interest in frontal volume/ cognition studies tend not to completely overlap with each other across studies, perhaps a better way to approach such questions would be to utilize complementary measures of brain structure (i.e. use additional measures such as both volume and thickness) to confirm the directionality of relationships. Taking these limitations into consideration, we briefly describe below our aims and methodology in undertaking a structure-function study of the frontal lobe.

\section{Current study}

The aim of the current study was to investigate structure-function relationships between the lateral frontal lobes and cognitive performance in healthy adults. A region of lateral frontal cortex was selected which completely overlaps with the dorsolateral prefrontal cortex (DLPFC) and includes other neighboring regions including the ventrolateral prefrontal cortex and the premotor cortex as these frontal regions show significant structural and functional changes with age (Raz et al., 2005; Tisserand et al., 2001). By utilizing large samples within narrow-age ranges and appropriate normalization of head sizes we intended to minimize age- and brain-size confounds so that structure-function correlations could be more stringently attributed to the region being investigated. An additional measure of cortical structure, cortical thickness, was used as an indicator of cerebral morphology as it can confirm volume results and provide an additional measure of cortical integrity. Furthermore, since structurefunction relationships have been shown to differ when participants are either young or old, we aimed to compare structure-function 
relationships in mid-life adults who typically have experienced less cognitive decline than older adults. Our study was therefore designed to investigate a) volume-cognition relationships in mid-life and older adults and b) thickness-cognition relationship in midlife and older adults.

We hypothesized that lateral frontal volumes would be associated with working memory and attention tasks. Given that healthy older adults have been found to show both positive and negative correlations in structure-function studies, we did not hypothesize a direction for this relationship. However, as older adults would presumably undergo more cognitive decline and age-related tissue loss than mid-life adults we hypothesized that frontal structurefunction associations would be stronger in older adults than mild-life adults. We also expected frontal thickness measures to have similar relationships as volume measures with cognition.

\section{Method}

\section{Subject selection}

Participants were recruited from the Personality and Total Health (PATH) Through Life Study. The PATH study consists of participants randomly recruited from the electoral roll living in Canberra in the ACT and the adjacent city of Queanbeyan. The project includes three age cohorts with individuals between the age group of 20-24, 40-44, and 60-64 who are followed up every four years. Participants were interviewed on cognitive function, sociodemographic variables, depression, anxiety and psychosocial risk factors. Further details on the PATH study have been published previously (Cherbuin et al., 2009; Christensen et al., 2009; Jorm et al., 2004).

This study concerns the second wave of data for the middle aged (MA) and early old-age (OA) participants, therefore they were between the ages of 44-48 and 64-68 for the current study. Due to the narrow-age range design, the participants are not fully representative of middle age and early old ages. Approval for the study was obtained from the human research ethics committee of the Australian National University and University of New South Wales. The MA cohort included 431 participants who completed the MRI scans. The OA cohort included in the study had 416 participants who underwent the MRI scan. For OA, 16 scans were excluded during image processing due to poor image quality, which made them unsuitable for automated analyses and 3 more participants were excluded due to incomplete cognitive data, so the total sample size was 397. Mean Mini Mental State scores for OA was 29.1. For the MA cohort, 1 scan was lost during acquisition and 30 scans were deemed unsuitable for semi-automated analyses and total sample size was 400 .

\section{Cognitive measures}

A cognitive battery was administered to the participants during the PATH interview preceding the MRI acquisition. Verbal working memory was measured through the Digits-Backwards span (DB), a subtest of the Wechsler memory scale (Wechsler, 1945), information processing speed and attention were measured through Symbol-Digit Modalities Test (SDM) (Smith, 1982), and mental flexibility and executive control were measured through Trail Making Tests A and B. Simple and choice reaction times were also measured and the procedure has been explained elsewhere (Mather et al., 2010). Episodic memory was measured with the first list of the California Verbal learning Test for both immediate and delayed recall (Delis et al., 1987). The cognitive tests utilized in the study are widely used in neuropsychological examinations and their test-retest reliabilities have been previously reported by other studies (for EM see Paolo et al., 1997, for DB see Iverson, 2001, for SDM see Hinton-Bayre and Geffen, 2005, and for Trails A and B see Cangoz et al., 2009). Missing data for cognitive measures were imputed using the EM imputation from SPSS.

In the interest of reducing the cognitive domains tested, simple and choice reaction times were combined using average z-scores to give reaction time (RT). Completion times for Trails $B$ and Trails A were used to create the ratio of Trails $\mathrm{B} /$ Trails $\mathrm{B}$ to give an index of mental flexibility (Drane et al., 2002; Oosterman et al., 2010). CVLT scores were highly correlated with each other $(r \sim 0.84)$ and were thus combined using z-scores to give an index of episodic memory (EM) to reduce multicollinearity. $\mathrm{Z}$ scores for $\mathrm{DB}$ and SDM were used as separate cognitive tests. Thus, in total five cognitive domains were tested: namely, episodic memory, processing speed, working memory, mental flexibility and reaction time.

\section{MRI acquisition}

MRI Acquisition parameters have been previously described in detail elsewhere (Cherbuin et al., 2009; Christensen et al., 2009). Briefly, participants were scanned on a 1.5 T Philips Gyroscan scanner (ACS-NT, Philips Medical Systems, Best, the Netherlands) for T1weighted 3D structural MRI in coronal orientation and Fast Field Echo sequence yielding contiguous slices with thickness of $1.5 \mathrm{~mm}$.

\section{Image analysis}

Images were transferred into a Linux workstation for analyses through a set of automated tools, FreeSurfer (http://surfer.nmr.mgh. harvard.edu/). FreeSurfer allows for automatic reconstruction of the cortical surface using T1-weighted MRI images. Major steps during image analyses include motion correction, averaging over multiple T1 images, removal of non-brain tissue, automated Talairach transformation, subcortical and cortical matter segmentation, intensity correction and delineation of grey/white/pial boundaries (Dale et al., 1999; Fischl et al., 1999). After the formation of cortical models, deformable procedures are applied including cortical inflation, registration to a spherical atlas and parcellation of the cerebral cortex into gyral and sulcal units (Desikan et al., 2006). Cortical thickness maps are created using both signal intensity and continuity information from the 3D-volume from MR images where thickness is calculated as the closest distance in from a pial to white matter boundary at each vertex (Dale et al., 1999). Reliabilities for cortical thickness measures obtained using FreeSurfer have been previously described (Han et al., 2006).

\section{Region selection}

After initial processing by FreeSurfer, all MRI scans were visually checked slice-by-slice to ensure there was no mis-registration of grey and white matter voxels; scans were reprocessed if errors were detected and rechecked visually a second time. Using the inbuilt atlas (Fischl et al., 2004), the volumes of the superior frontal, middle frontal and inferior frontal gyri were imported from FreeSurfer. The boundaries for superior frontal region were the superior frontal sulcus rostrally, the paracentral sulcus caudally and the medial aspects of the frontal lobe and superior frontal sulcus laterally (Fig. 1).The middle frontal gyral region included the area of the cortex that was bound by the superior frontal sulcus rostrally, the precentral gyrus caudally, and the inferior frontal gyrus and the medial aspect of middle frontal gyrus laterally. The inferior frontal region included the area of the cortex within the regions bounded by the inferior frontal sulcus rostrally, the precentral gyrus caudally and the laterally and medially by the inferior frontal sulcus and lateral orbital sulcus respectively. A composite volume of the lateral frontal cortex was calculated by adding the volumes of superior, middle and inferior frontal gyri of both hemispheres. The frontopolar region and 


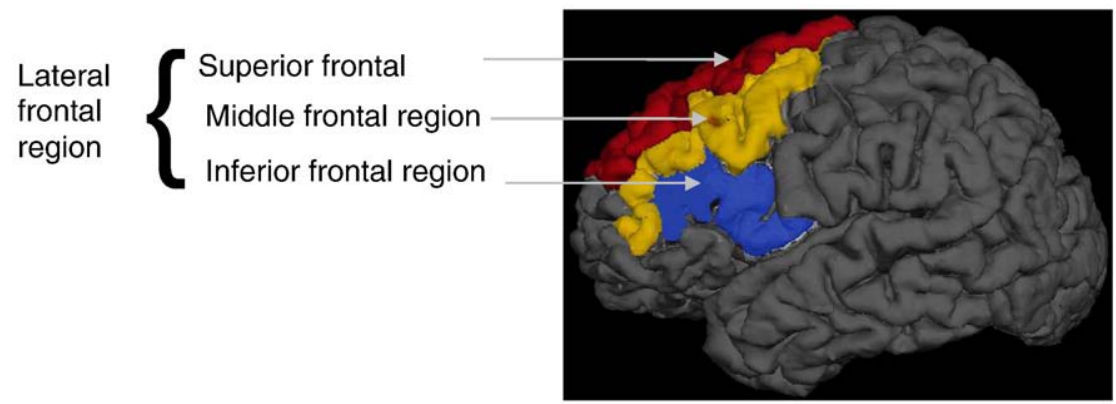

Fig. 1. Lateral frontal cortex and its component regions.

the orbitofrontal regions were not included for the lateral frontal volumes.

\section{Statistical analyses}

Analyses were conducted in PASW Statistics 18 and AMOS 18. Zero order correlations with cognitive variables and frontal cortex were estimated for descriptive purposes. Two different sets of statistical analyses were conducted for the two cortical measures of volume and thickness: namely, path analysis for volume-cognition relationships and general linear modeling (GLM) for thickness-cognition relationships. Path analyses and GLM analyses were conducted separately for the two age cohorts.

Scatterplots for the distribution of male and female participants in the two cohorts are given in Fig. 5. Females had better performance than males in EM, SDM and DB performance in OA adults only, but this difference was not statistically significant after controlling for the effects of age, education and ICV.

\section{Volume-cognition relationships}

A latent variable for cognition was not used because the aim of the analysis was to investigate specific relationships between cognitive measures and brain volumes. Variables were centered on their means and models were fitted using maximum likelihood methods based on multivariate normal assumptions. The models were constructed with education, gender, age and TBV (Total Brain Volume) considered independent variables which along with the LFC were antecedent to the cognitive variables (dependent variables). Volumes of LFC and TBV were normalized against ICV before being entered into the model to control for variance due to head size. Additionally, as age-related atrophy would be different in MA and OA groups, we also included TBV when investigating the structure function relationships. Similarly, education and gender were added as independent variables as they have also been shown to be predictive of cognitive function. Therefore, age, TBV, gender and education were considered the first tier of the model out of which age, gender and TBV were presumed to affect the second tier variable, LFC. All the independent variables along with LFC were then presumed to affect the third tier cognitive variables.

During path analyses, a statistically significant chi-squared value can spuriously occur when sample sizes are large; therefore, other measures of fit indices are preferred for indications of goodness of fit. A value for the comparative fit index $(\mathrm{CFI})>0.95$ and a value of rootmean square error of approximation (RMSEA) $<0.05$ are considered to demonstrate a good fit for the data. Similarly, a smaller Akaike information criteria (AIC) value rather than the absolute value is preferred when nested models are compared. Goodness of fit in our analyses was obtained through comparisons of CFI, AIC as well as RMSEA values for each model. After setting the initial model (described in previous paragraph), the subsequent models were

Table 1

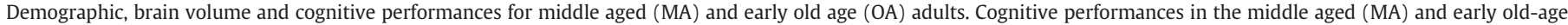
(OA) adults after controlling for education and gender.

\begin{tabular}{|c|c|c|c|c|c|c|}
\hline & \multicolumn{2}{|l|}{ MA } & \multicolumn{2}{|l|}{$\mathrm{OA}$} & \multirow[t]{2}{*}{ F value } & \multirow[t]{2}{*}{$\mathrm{p}$} \\
\hline & Mean & SD & Mean & SD & & \\
\hline Age in years & 46.7 & 1.40 & 66.5 & 1.40 & & \\
\hline Education in years (range) & $14.6(9-18)$ & 2.20 & $14.1(4-18)$ & 2.60 & 8.25 & 0.004 \\
\hline Females (\%) & 222 & & 177 & & 7.79 & 0.005 \\
\hline English as first language (\%) & 386 & & 359 & & 5.58 & 0.018 \\
\hline Hypertension (\%) & $56(14.1 \%)$ & & $187(47 \%)$ & & 35.81 & 0.0001 \\
\hline Diabetes (\%) & $8(2 \%)$ & & $40(10 \%)$ & & 108.41 & 0.0001 \\
\hline ICV & $1578 \mathrm{~cm}^{3}$ & 154 & $1533 \mathrm{~cm}^{3}$ & 178 & 1.41 & 0.231 \\
\hline TBV & $1073 \mathrm{~cm}^{3}$ & 109 & $1003 \mathrm{~cm}^{3}$ & 105 & 81.21 & 0.0001 \\
\hline Lateral frontal (LFC) & $75.36 \mathrm{~cm}^{3}$ & 8.70 & $68.96 \mathrm{~cm}^{3}$ & 8.00 & 301.2 & 0.001 \\
\hline Superior frontal & $40.32 \mathrm{~cm}^{3}$ & 4.80 & $37.56 \mathrm{~cm}^{3}$ & 4.40 & 139.4 & 0.001 \\
\hline Middle frontal & $22.32 \mathrm{~cm}^{3}$ & 3.20 & $20.93 \mathrm{~cm}^{3}$ & 3.10 & 58.91 & 0.001 \\
\hline Inferior frontal & $12.75 \mathrm{~cm}^{3}$ & 1.69 & $10.46 \mathrm{~cm}^{3}$ & 1.40 & 579 & 0.001 \\
\hline Immediate recall & 8.26 & 2.20 & 6.97 & 2.00 & 24.11 & 0.0001 \\
\hline Delayed recall & 7.57 & 2.40 & 6.07 & 2.30 & 24.00 & 0.0001 \\
\hline Digit backwards & 5.83 & 2.20 & 5.11 & 2.10 & 5.00 & 0.0250 \\
\hline Symbol-digit modalities & 61.21 & 8.60 & 50.35 & 8.69 & 147.7 & 0.0001 \\
\hline Trails A (time seconds) & 24.95 & 7.13 & 34.2 & 10.7 & 36.03 & 0.0001 \\
\hline Trails B (time s) & 55.6 & 17.9 & 79.5 & 29.1 & 56.31 & 0.0001 \\
\hline Choice reaction (time s) & 0.29 & 0.03 & 0.32 & 0.05 & 25.63 & 0.0001 \\
\hline Simple reaction (time s) & 0.23 & 0.06 & 0.27 & 0.06 & 18.01 & 0.0001 \\
\hline
\end{tabular}


made parsimonious by removing non-significant paths at a 0.1 level. The resulting model was then made more parsimonious by removing other non-significant paths after the first step.

\section{Thickness-cognition analyses using GLM}

Structure-function relationships using frontal cortical thickness maps and cognitive measures were investigated where the LFC was the region of interest. Thickness maps were spatially smoothed with a Gaussian kernel with a half maximum width of $10 \mathrm{~mm}$. Maps were then averaged across participants using a spherical aligning method for cortical folding patterns (Fischl et al., 1999). Using GLM, thickness measures were correlated with cognitive performance using in-built statistical analyses engine in FreeSurfer to identify significant clusters on the cortical surface. Surface analyses were conducted for each hemisphere separately. Corrections for multiple comparisons were done through two methods: firstly, LFC was chosen as region of interest which limited the number of comparisons. Secondly, since permutation testing is a more robust form of detection of cluster-wise significance, thickness maps were smoothed iteratively (1000 iterations) to derive an estimation threshold for significant clusters at $\mathrm{p}<0.01$.

\section{Results}

The ICV, raw volumes of the frontal regions and total lateral frontal volumes for both cohorts are given in Table 1. Analyses using ANOVA showed that there were no differences in ICV between the two age groups, $(p<0.231, F=1.45, d f=1)$, while $T B V$ was significantly smaller for OA than MA $(\mathrm{p}<0.0001, \mathrm{~F}=81, \mathrm{df}=1)$. All regional volumes and hence the total LFC volume were significantly lower in the OA compared with MA $(\mathrm{p}<0.001, \mathrm{~F}=309.4, \mathrm{df}=1)$. The MA cohort performed significantly better than the OA cohort in all of the cognitive tests, after adjusting for education and sex as shown in Table 1.

Zero order correlations for all the cognitive variables in the model are presented in Tables $2(\mathrm{OA})$ and $3(\mathrm{MA})$. For OA, significant associations were observed between LFC volumes and cognitive measures (EM and DB) as well as ICV, and TBV. Similar associations between brain variables, LFC and DB were found for MA.

\section{Volume-cognition relationships}

In the path analyses, the starting model had a model fit of: $\chi^{2}=55.6$, $\mathrm{p}=0.001, \mathrm{CFI}=0.945$, RMSEA $=0.051$, and AIC $=279.6$. This model was successfully reduced by removing correlations with significance higher than 0.1 resulting with model fit values of: $\chi^{2}=49.9, p=0.006$, $\mathrm{CFI}=0.978$, RMSEA $=0.031$, and AIC $=253.9$. After controlling for TBV, the effects of age on frontal cortex as well as the cognitive tests were non-significant, so the non-significant paths were removed from the final model and this further significantly improved the model fit: $\chi^{2}=44.8, p=0.066, \mathrm{CFI}=0.983, \mathrm{RMSEA}=0.023$, and $\mathrm{AIC}=196.8$. The final parsimonious model is presented in Fig. 2 and the standardized regression estimates for the associations between cognitive measures and brain variables are shown in Table 4.

Significant relationships between education and the cognitive variables were found for MA (Table 4). Significant standard regression estimates were $\mathrm{DB}=0.162, \mathrm{p}<0.001$, and $\mathrm{EM}=0.138, \mathrm{p}<0.005$. While the zero order correlation for LFC was significantly associated with DB scores, after controlling for variability due to brain size using ICV, this association became non-significant.

For OA, all cognitive measures except RT were significantly associated with education while all cognitive indices except EM were significantly associated with TBV (Table 4). EM $(-0.117$, $\mathrm{p}<0.012)$ as well as SDM $(-0.118, \mathrm{p}<0.030)$ was found to be negatively associated with LFC, (standardized estimates and p values in brackets). The significant relationship between LFC and SDM is only revealed after including TBV into the model which suggests that TBV acted as a suppressor variable in OA, Table 5. Interactions between TBV and LFC with SDM as an independent variable were tested with ANOVA but were found to be non-significant $(\mathrm{df}=1, \mathrm{~F}=0.54$, $\mathrm{p}=0.46)$.

\section{Thickness-cognition relationships}

Associations between cognitive measures and frontal cortical thickness were first evaluated and then repeated after progressively controlling for each independent variable in the following order: ICV, age, education and gender. Controlling for any of the independent variables did not make significant differences to the relationships. Significant associations with cognitive indices with the frontal cortex were found in both the OA (Fig. 3) and MA cohorts (Fig. 4).

For OA, cortical thickness of regions of the lateral frontal cortex was significantly negatively associated with SDM $(p<0.01)$ in the right hemisphere and with EM $(p<0.01)$ in both hemispheres. None of the other cognitive measures survived the significance testing for clusters at $\mathrm{p}<0.01$ for $\mathrm{OA}$. In MA, EM scores $(\mathrm{p}<0.01)$ showed a positive relationship with cortical thickness in both hemispheres while RT $(p<0.01)$ scores were significantly correlated with thickness in the left hemisphere. TAB scores $(p<0.05)$ were marginally significant.

To compare the thickness model with the volume path analyses model, additional analyses were undertaken using mean thickness values of the three frontal regions, namely superior frontal, middle frontal and inferior frontal regions in LFC. The final results for these analyses were comparable with results from 2.6.1 where significant relationships were found between SDM and EM in the middle frontal

Table 2

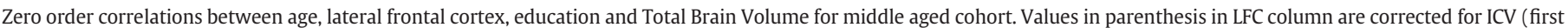

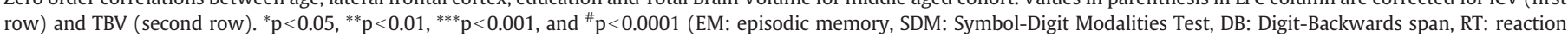

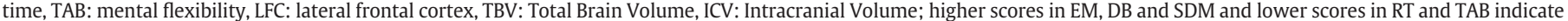
better performance).

\begin{tabular}{|c|c|c|c|c|c|c|c|c|}
\hline & Age & EM & SDM & DB & RT & $\mathrm{TAB}$ & LFC & TBV \\
\hline EM & $0.106^{*}$ & & & & & & & \\
\hline SDM & $-0.121^{*}$ & $0.295^{\#}$ & & & & & & \\
\hline DB & 0.051 & $0.209^{\#}$ & $0.207^{\#}$ & & & & & \\
\hline RT & 0.071 & -0.026 & $-0.282^{\#}$ & $-0.129 *$ & & & & \\
\hline TAB & 0.061 & $-0.19^{\#}$ & $-0.402^{\#}$ & $-0.282^{\#}$ & $0.192^{\#}$ & & & \\
\hline \multirow[t]{3}{*}{ LFC } & -0.075 & -0.068 & 0.063 & $0.159^{* *}$ & $-0.131^{*}$ & -0.082 & & \\
\hline & $(-0.051)$ & $(-0.051)$ & $(0.059)$ & $(0.041)$ & $(0.029)$ & $(0.036)$ & & \\
\hline & $(0.001)$ & $(0.026)$ & $(0.001)$ & $(0.015)$ & $(0.052)$ & $(-0.024)$ & & \\
\hline TBV & -0.092 & -0.085 & 0.083 & $0.175^{* * *}$ & $-0.178^{\#}$ & -0.088 & $0.872^{\#}$ & \\
\hline Education & -0.071 & $0.106^{*}$ & $0.160^{* *}$ & $0.162^{* *}$ & -0.090 & -0.096 & $0.172^{* *}$ & $0.164^{* *}$ \\
\hline
\end{tabular}


Table 3

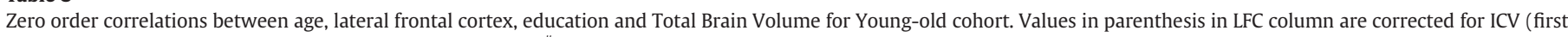

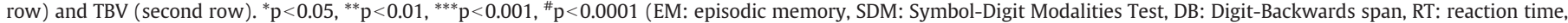

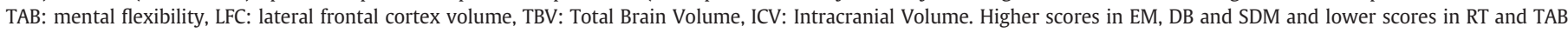
indicate better performance).

\begin{tabular}{|c|c|c|c|c|c|c|c|c|}
\hline & Age & EM & SDM & DB & RT & $\mathrm{TAB}$ & LFC & TBV \\
\hline EM & 0.076 & & & & & & & \\
\hline SDM & 0.012 & $0.194^{\#}$ & & & & & & \\
\hline DB & -0.018 & $0.188^{\#}$ & $0.412^{\#}$ & & & & & \\
\hline RT & 0.018 & $-0.173^{* * * *}$ & $-0.23^{\#}$ & $-0.108^{*}$ & & & & \\
\hline TAB & -0.042 & -0.071 & $-0.373^{\#}$ & $-0.372^{\#}$ & 0.013 & & & \\
\hline \multirow[t]{3}{*}{ LFC } & -0.044 & $-0.247^{\#}$ & 0.040 & $0.146^{* *}$ & -0.055 & -0.094 & & \\
\hline & $(-0.100)$ & $\left(-0.162^{* * *}\right)$ & $(-0.019)$ & $(0.054)$ & $(0.043)$ & $(-0.054)$ & & \\
\hline & $(0.037)$ & $\left(-0.179^{* *}\right)$ & $\left(-0.147^{* *}\right)$ & $(-0.072)$ & $(0.098)$ & $(0.034)$ & & \\
\hline TBV & -0.075 & $-0.191^{\#}$ & $0.126^{*}$ & $0.209^{\#}$ & $-0.117^{*}$ & -0.132 & $0.874^{\#}$ & \\
\hline Education & -0.004 & $0.206^{\#}$ & $0.229^{\#}$ & $0.259^{\#}$ & -0.085 & $-0.129 *$ & $0.196^{\#}$ & $0.200^{\#}$ \\
\hline
\end{tabular}

region in $\mathrm{OA}$ (negative associations). Similarly, for MA, relationships between cognitive measures and frontal cortical thickness were mixed with significant relationships between EM in middle frontal and RT in superior frontal regions (positive associations). Values for the final model fit, standard regression estimates and the final path model have been provided in supplementary analyses (Supplementary figure, Tables 6 and 7).

\section{Participants with mild cognitive disorders}

Due to the age of the participants, it is possible that the OA cohort included some with incipient cognitive impairment, influencing the findings. Therefore, we further re-examined the data by excluding participants who had any sort of memory impairment diagnoses upon further testing. The procedures for the diagnoses have been explained elsewhere (Anstey et al., 2008). Older adults who had any kind of mild cognitive disorders including $\mathrm{MCI}$ were excluded $(n=15)$ and data were re-analyzed. There were no significant differences in the findings with and without the participants with mild cognitive disorders.

\section{Discussion}

\section{Main findings}

Significant negative associations were found between episodic memory (EM, measured using the immediate and delayed recall scores from the California verbal learning test), Symbol-Digit Modalities Task (SDM), and the lateral frontal cortex in the OA adults from path analyses investigating volume/function relationships. Correlations of cortical thinning with EM and SDM scores in OA participants confirmed negative associations. In contrast, no significant associations were found between any of the cognitive tests and LFC volume for MA adults while frontal cortical thickness was positively associated with EM and reaction times (RT). Since OA adults displayed significant relationships both with volume and thickness measures, our hypothesis that OA adults would display stronger structure-function relationships with cognition was supported. Pairwise parameter estimates comparisons in path analyses also showed that regression coefficients for EM and SDM were significantly different for MA and OA adults, (data not shown). Thus, in $\mathrm{OA}$, smaller or less preserved frontal cortices were associated with

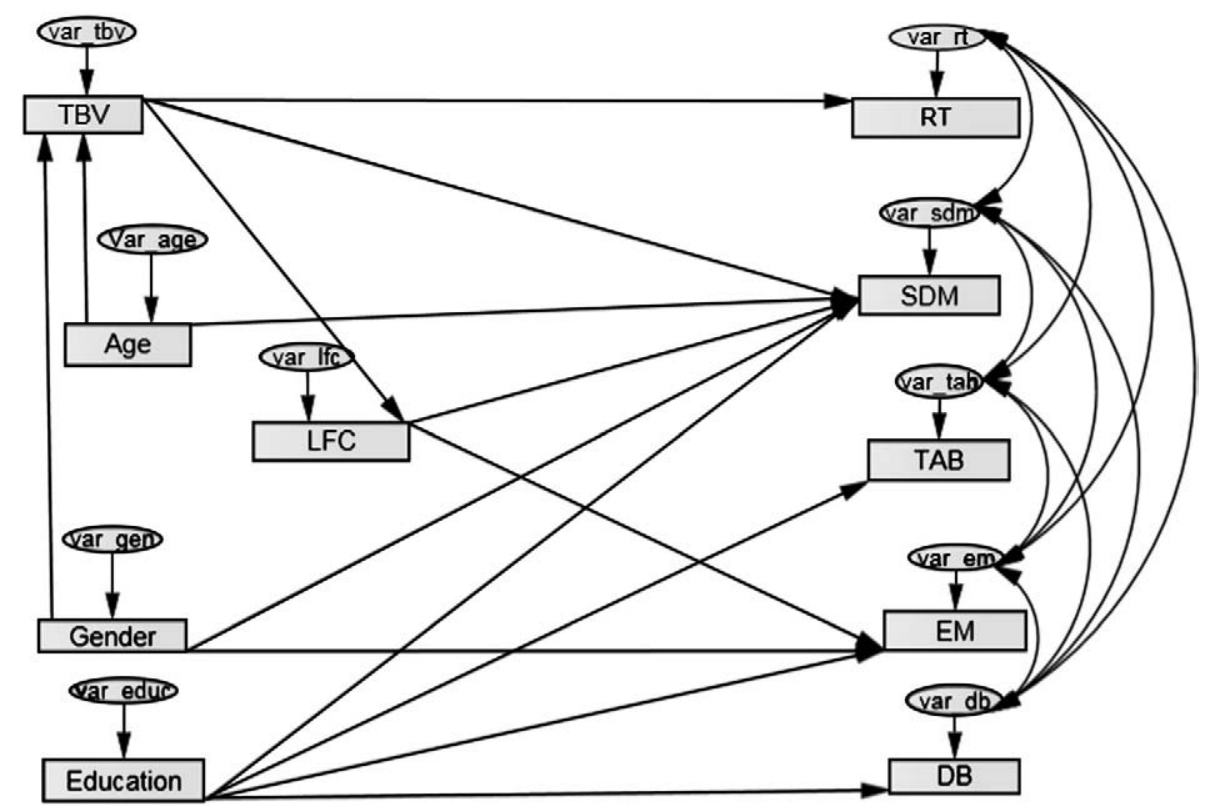

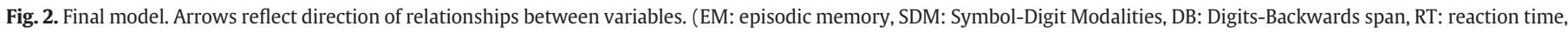

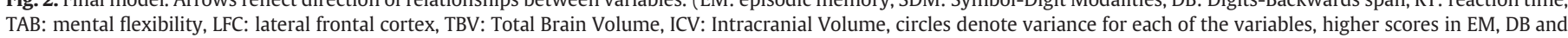
SDM and lower scores in RT and TAB indicate better performance.) 
Table 4

Standardized regression coefficients from SEM analyses for middle aged (MA) and early old-age (OA) adults for the final model. ${ }^{*} \mathrm{p}<0.05$, ${ }^{* *} \mathrm{p}<0.01$, and ${ }^{* * *} \mathrm{p}<0.001$ (EM: episodic memory, SDM: Symbol-Digit Modalities Test, DB: Digit-Backwards span, RT: reaction time, TAB: mental flexibility, LFC: lateral frontal cortex, TBV: Total Brain Volume, ICV: Intracranial Volume; higher scores in EM, DB and SDM and lower scores in RT and TAB indicate better performance).

\begin{tabular}{|c|c|c|}
\hline \multirow[t]{2}{*}{ Paths } & Sd. regression coefficients & Sd. regression coefficients \\
\hline & MA & $\mathrm{OA}$ \\
\hline $\mathrm{LFC} \rightarrow \mathrm{EM}$ & 0.048 & $-0.181^{* * *}$ \\
\hline $\mathrm{LFC} \rightarrow \mathrm{SDM}$ & -0.033 & $-0.187^{*}$ \\
\hline $\mathrm{TBV} \rightarrow \mathrm{SDM}$ & $0.197^{*}$ & $0.309^{* *}$ \\
\hline Age $\rightarrow$ TBV & -0.070 & $-0.165^{* * *}$ \\
\hline Education $\rightarrow$ EM & $0.126^{* *}$ & $0.264^{* * *}$ \\
\hline Education $\rightarrow$ DB & $0.133^{* *}$ & $0.225^{* * *}$ \\
\hline Education $\rightarrow$ SDM & $0.141^{* *}$ & $0.214^{* * *}$ \\
\hline Education $\rightarrow \mathrm{TAB}$ & -0.073 & $-0.109^{*}$ \\
\hline Gender $\rightarrow$ TBV & 0.041 & $0.177^{* * *}$ \\
\hline
\end{tabular}

better cognitive performance thereby suggesting that for our sample during healthy aging, smaller frontal volumes were associated with better cognitive performance.

Additionally, Total Brain Volume (TBV) was significantly associated with DB (Digit-Backwards span), SDM, TAB and RT in OA with TBV acting as a suppressor for the relationship between LFC and SDM. This effect of TBV was not seen in any other cognitive domain in OA or in MA although significant zero-order correlations were found between TBV and DB, SDM and RT in MA.

\section{Significance of findings}

Cognitive performance and frontal volumes have been found to be different for mid-life and older adults and are not completely explained by age (Raz et al., 2005; Salthouse, 2003; Tisserand et al., 2001) as observed in the present findings. Our results are also consistent with other studies that show that lateral frontal cortices are important in frontally mediated functions such as attention, visual scanning and verbal recall (Miller and Cohen, 2001). The direction of associations between the two age groups differed with OA showing consistent negative relationships while MA showed mixed relationships (positive for EM and negative for RT) which suggests that different relationships between cognitive function and frontal cortical structure occur at different ages. A similar difference in lateral frontal volume and its age-dependent change in the relationship with executive functions has previously been described (Zimmerman et al., 2006). The positive relationship between frontal cortical thickness and episodic memory in MA is concordant with a large majority of structure-function studies that have studied this relationship in the frontal cortex (Elderkin-Thompson et al., 2008; Köhler et al., 1998; Müller and Knight, 2006; Raz et al., 1999).

Table 5

Associations between Symbol Digit Modalities scores (SDM) and Lateral Frontal cortex (LFC) volumes with suppressor Total Brain Volume (TBV) ( ${ }^{*}$ correlations significant at $\mathrm{p}<0.01$ level) (Higher scores in SDM and indicate better performance.)

\begin{tabular}{lcccc}
\hline Volumes & \multicolumn{4}{l}{ Correlations with SDM scores } \\
\cline { 2 - 5 } & Beta weights & Zero order & Semi-partial & Partial \\
\hline${ }^{1}$ LFC & 0.042 & 0.042 & 0.042 & 0.042 \\
${ }^{2}$ TBV, LFC & $-0.309^{\#}$ & 0.042 & $-0.161^{\#}$ & $-0.159^{\#}$ \\
${ }^{3}$ TBV, LFC & $-0.150^{\#}$ & -0.069 & $-0.149^{\#}$ & $-0.150^{\#}$ \\
\hline
\end{tabular}

Note: Hierarchical linear regression analyses with SDM scores as dependent variable with.

${ }^{1}$ LFC volume as independent variable.

${ }^{2}$ TBV volume entered first followed by LFC as independent variable.

${ }^{3}$ Volumes normalized against Intracranial Volumes before entering into model, TBV entered first followed by LFC volume.

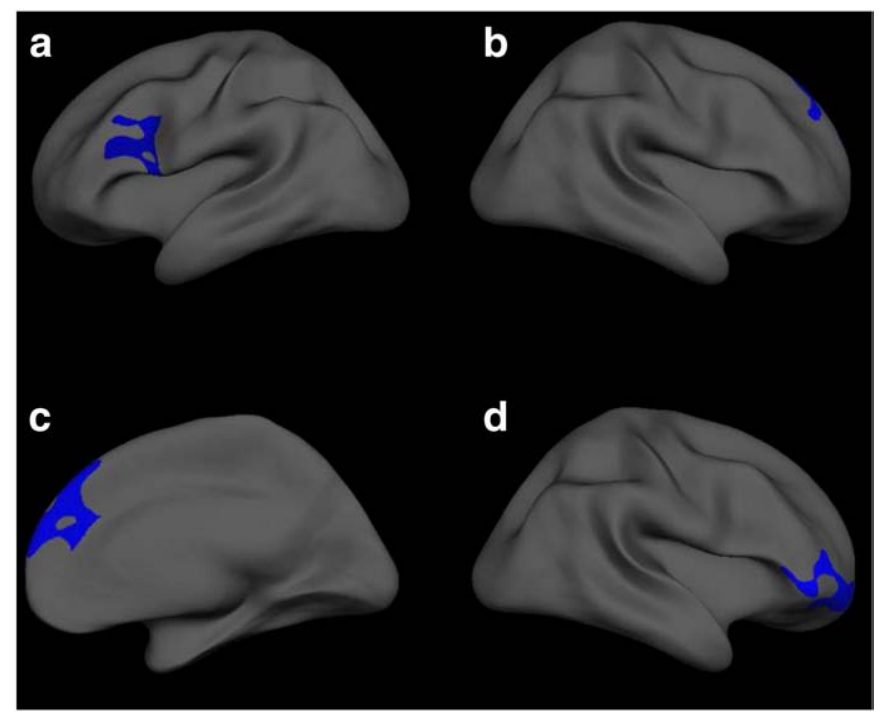

Fig. 3. Correlations between cognitive indices and thickness maps for early old-age (OA) adults: a) episodic memory LH lateral view, b,c) episodic memory RH lateral and medial view and d) Symbol-Digit Modalities RH. Blue indicates a negative correlation, clusters significant at $\mathrm{p}<0.01$. LH: left hemisphere, $\mathrm{RH}$ : right hemisphere. (For interpretation of the references to color in this figure legend, the reader is referred to the web version of this article.)

Regarding the direction of (or lack thereof) associations in OA, our results are also consistent with a number of studies that have found null (Shenkin et al., 2003; Staff et al., 2004) and negative relationships (Duarte et al., 2006; Köhler et al., 1998; Salat et al., 2002; Van Petten et al., 2004) between grey matter volumes and neuropsychological tests in studies of healthy adults - and are in contrast to studies that have reported positive correlations during $\mathrm{AD}$ and $\mathrm{MCl}$ (Rossi et al., 2007; Van Der Flier et al., 2002). The idea that losses of cortical volumes in neuropathology have different effects on cognition is not a

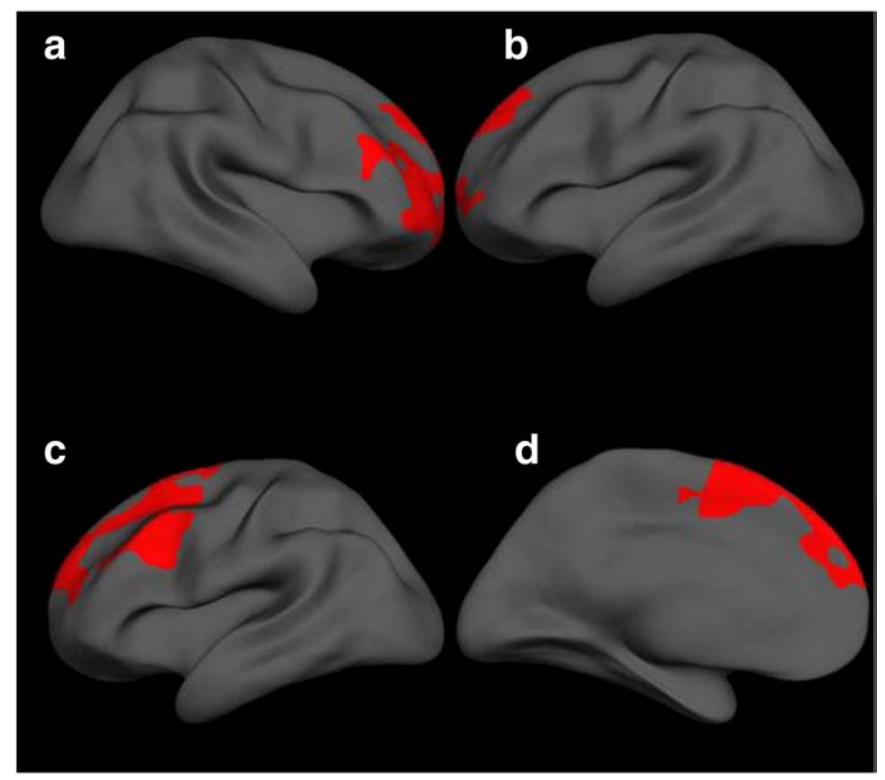

Fig. 4. Correlations between cognitive indices and frontal thickness maps for middle aged (MA) cohort: a,b) episodic memory, $\mathrm{RH}$ and $\mathrm{LH}$ and c,d) reaction time, LH lateral and medial views. Red indicates a positive correlation, clusters significant at $\mathrm{p}<0.01$. $\mathrm{LH}$ : left hemisphere, RH: right hemisphere. (For interpretation of the references to color in this figure legend, the reader is referred to the web version of this article.) 
new one as numerous studies show that the cognitive profiles and neuronal mechanisms involved are very different from normal aging (Baxter et al., 2006; Braak and Braak, 1997). A study by Duarte et al. (2006) specifically investigated these differences by correlating frontal and temporal lobe volumes with cognitive performance in healthy older adults and adults with $\mathrm{AD}$ and found that adults with $\mathrm{AD}$ had positive relationships while healthy older adults had negative structure-function relationships. OA adults in the present study are of similar ages and cognitive profile to the study by Duarte et al. (2006); additionally, while this and other previous studies have investigated structure-function associations with just volume measures, we have found complimentary relationships using both volume as well as thickness measures. Our findings thus argue against spurious associations between variables. However, (as pointed out by an anonymous reviewer), both cortical measures have been calculated using similar pre-processing steps and thus could be subject to the same biases in measurement.

While negative relationships were found between frontal volumes, significant positive relationships were found with TBV and cognitive measures. TBV was positively and significantly correlated with LFC and SDM, additionally acting as a suppressor in the relationship between LFC and SDM. Analyses of regression weights, partial and semi-partial correlations show that predictive value of LFC in SDM increases and becomes significant after controlling for TBV from a previously non-significant correlation (Table 5) (Conger, 1974). This contribution of TBV indicates that including it in the model reduces the irrelevant variance in LFC in its relationship with SDM. The identification of TBV as a suppressor highlights the importance of identifying possible predictor variables in a structure-function relationship and how inadvertent exclusion of such variables might

\section{Middle aged (MA)}
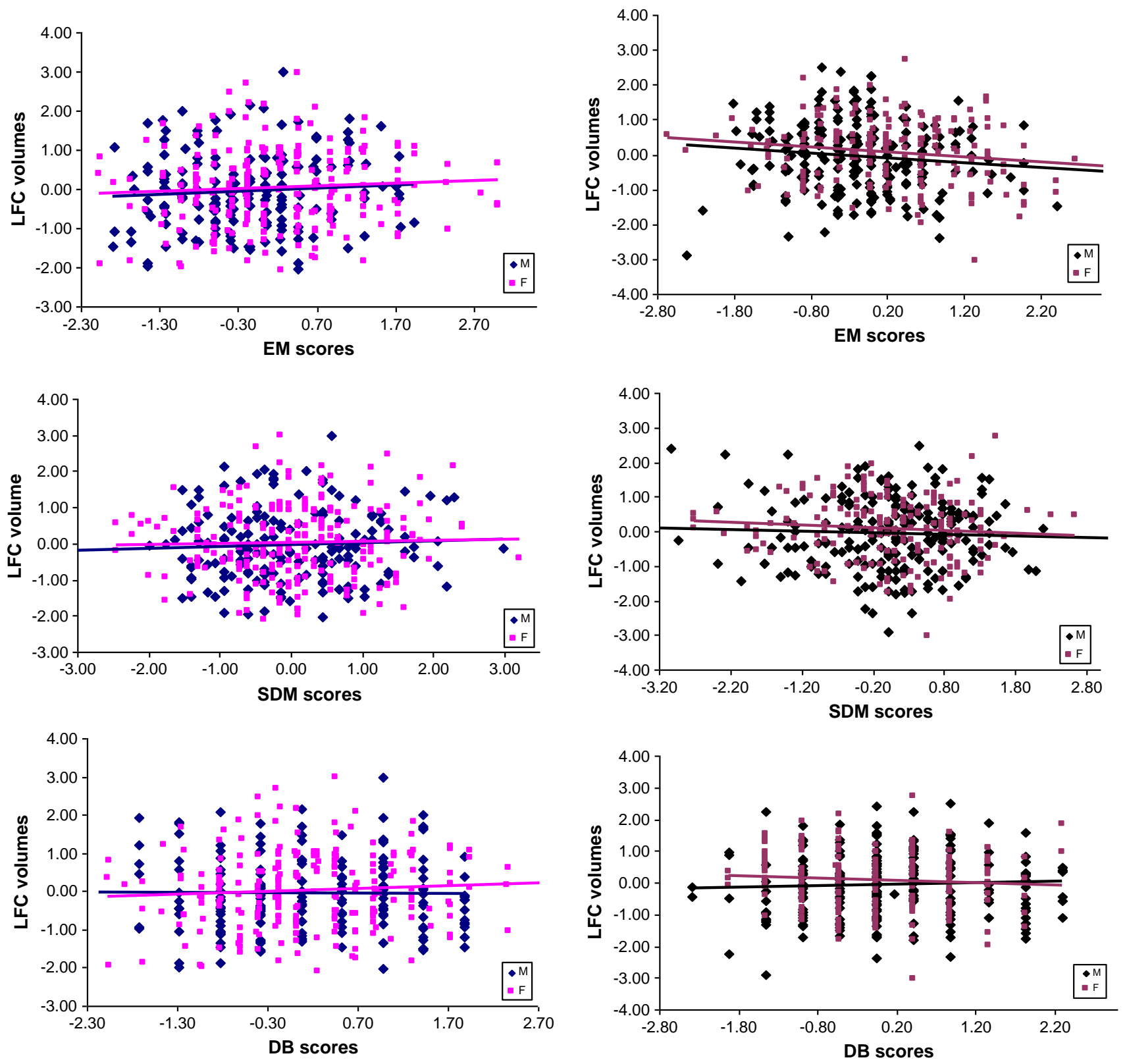

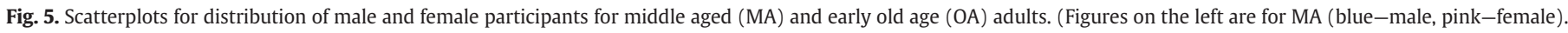

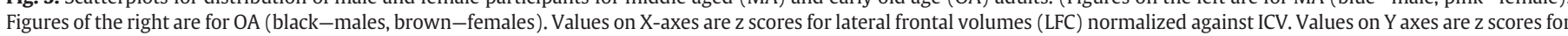
the cognitive indices.) (For interpretation of the references to color in this figure legend, the reader is referred to the web version of this article.) 

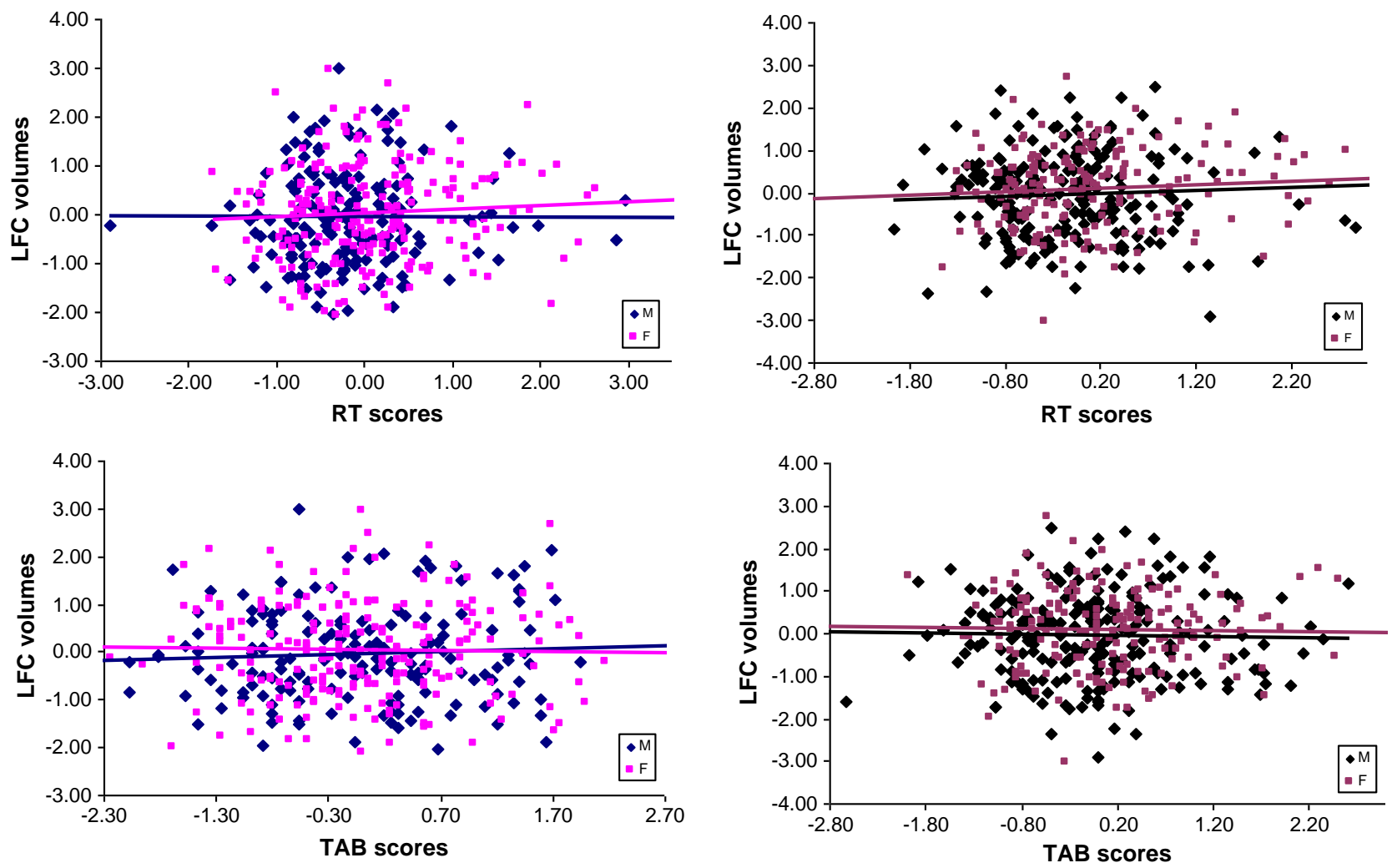

Fig. 5. (continued).

obscure structure-function relationships resulting in apparently null relationships. It is also worthwhile thinking about whether there might be other related variables that might improve the relationship between LFC and other variables that we have not included in the model.

In addition to a possible inhibitory role of the frontal cortices in episodic retrieval as mentioned in the Age and frontal structurefunction relationship in aging section, a likely inhibitory role of the frontal cortex in cognition was also seen in a recent study which showed that conflict-resolution by rats with lesions in the middle frontal cortex was equivalent to that by sham-lesioned animals (Dwyer et al., 2009). The subsequently smaller functional region in this case was thought to reduce response-conflict by affecting input of interfering information. This also emphasizes the notion of neural efficiency in older adults especially in the context age-related volume decline. Efficient brain structures are detected by negative correlations during task activations (Solé-Padullés et al., 2009; Stern et al., 2005) and so, with consideration to neural communication, just as in development, smaller volumes might be desirable in older age if it denotes highly functional cortical connections.

Lastly, if the decline in cognitive processing begins before measurable changes in volume, then volume-cognition associations would not have a proportional relationship. The best way to test for these relationships would be to follow participants longitudinally and assess the structural and cognitive decline with time. Results from some of the published studies so far have been mixed. Rodrigue and Raz (2004) found that after 5 years, the change in volumes of neither the prefrontal cortex nor the hippocampus was associated with memory decline, however, the change in volume of the entorhinal cortex was. However, other studies (Burgmans et al., 2010; Tisserand et al., 2004), did find that changes in prefrontal volume predicted cognitive decline, but the cognitive measures used and the sample characteristics were too different to make a proper comparison. Our results should therefore be cautiously interpreted until further longitudinal follow-up is carried out.

\section{Limitations}

Our study had a number of strengths over other studies conducted so far on structure-function relations of the lateral frontal cortex. We utilized epidemiological samples, and the cohorts utilized in this study have been selected from a very narrow age range so potential age-confounding effects have been limited. We have simultaneously studied structure-function relationships using brain volumes and thickness and this is to our knowledge the first study to do so in the same sample and underscores the importance of utilizing various cortical morphometry measures in studying structure-function associations in normal aging adults.

However, despite the large sample sizes, our narrow-age design could have restricted normal statistical variance of the sample studied. Also, being a cross-sectional study, the causal nature of the differences we have observed cannot be inferred and need confirmation from longitudinal findings. This design also has limitations in controlling for inter-individual differences and cohort effects in the sample and can underestimate true change (Sliwinski and Buschke, 1999) and thus any pre-existing cognitive differences if they existed in the two cohorts could not be controlled for. We also had limited cognitive tests available to us to assess the structure-function relationships and which restricted the cognitive domains investigated.

\section{Conclusions}

In summary, we have found using large epidemiological samples that frontal structure-function relationships are different for middle aged and older adults. We also found that in older adults, lateral 
frontal brain volumes and cortical thickness correlate similarly with cognitive measures and that smaller frontal volume during older age is not necessarily associated with poorer cognitive performances. As previous reports of structure-function associations have been mixed, further longitudinal analyses are warranted to study changes in cognition with age.

\section{Acknowledgments}

The authors are grateful to Naftali Raz, Anthony Jorm, Bryan Rodgers, Helen Christensen, Chantal Reglade-Meslin, Patricia Jacomb, Karen Maxwell, Andrew Janke, and the PATH interviewers. This research was supported by National Health and Medical Research Council (NHMRC) of Australia Unit Grant No. 973302, Program Grant No. 179805, Project Grant No. 157125, grants from the Australian Rotary Health Research Fund and the Australian Brewers Foundation, and a processing grant from the National Computational Infrastructure. Nicolas Cherbuin is funded by NHMRC Research Fellowship No. 471501. Kaarin Anstey is funded by NHMRC Research Fellowship No. 366756

\section{Appendix A. Supplementary data}

Supplementary data to this article can be found online at doi:10.1016/j.neuroimage.2011.01.015.

\section{References}

Anstey, K.J., Cherbuin, N., Christensen, H., Burns, R., Reglade-Meslin, C., Salim, A., Kumar, R., Jorm, A.F., Sachdev, P., 2008. Follow-up of mild cognitive impairment and related disorders over four years in adults in their sixties: the PATH Through Life Study. Dement. Geriatr. Cogn. Disord. 26, 226-233.

Bartrés-Faz, D., Solé-Padullés, C., Junqué, C., Rami, L., Bosch, B., Bargalló, N., Falcón, C. Sánchez-Valle, R., Molinuevo, J.L., 2009. Interactions of cognitive reserve with regional brain anatomy and brain function during a working memory task in healthy elders. Biol. Psychol. 80, 256-259.

Baxter, L.C., Sparks, D.L., Johnson, S.C., Lenoski, B., Lopez, J.E., Connor, D.J., Sabbagh, M.N., 2006. Relationship of cognitive measures and gray and white matter in Alzheimer's disease [see comment] J. Alzheimers Dis. 9, 253-260.

Braak, H., Braak, E., 1997. Frequency of stages of Alzheimer-related lesions in different age categories. Neurobiol. Aging 18, 351-357.

Buckner, R.L., 2004. Memory and executive function in aging and AD: multiple factors that cause decline and reserve factors that compensate. Neuron 44, 195-208.

Burgmans, S., van Boxtel, M.P.J., Smeets, F., Vuurman, E.F.P.M., Gronenschild, E.H.B.M., Verhey, F.R.J., Uylings, H.B.M., Jolles, J., in press. Prefrontal cortex atrophy predicts dementia over a six-year period. Neurobiology of Aging 30, 1413-1419.

Cangoz, B., Karakoc, E., Selekler, K., 2009. Trail Making Test: normative data for Turkish elderly population by age, sex and education. J. Neurol. Sci. 283, 73-78.

Chantôme, M., Perruchet, P., Hasboun, D., Dormont, D., Sahel, M., Sourour, N., Zouaoui, A., Marsault, C., Duyme, M., 1999. Is there a negative correlation between explicit memory and hippocampal volume? Neuroimage 10, 589-595.

Cherbuin, N., Anstey, K.J., Reglade-Meslin, C., Sachdev, P.S., 2009. In vivo hippocampal measurement and memory: a comparison of manual tracing and automated segmentation in a large community-based sample. PLoS ONE 4, e5265 (Electronic Resource).

Christensen, H., Batterham, P.J., Mackinnon, A.J., Anstey, K.J., Wen, W., Sachdev, P.S., 2009. Education, atrophy, and cognitive change in an epidemiological sample in early old age. Am. J. Geriatr. Psychiatry 17, 218-226 (March).

Conger, A., 1974. A revised definition for suppressor variables: a guide to their identification and interpretation. Educ. Psychol. Meas. 34, 35-46.

Courchesne, E., Chisum, H.J., Townsend, J., Cowles, A., Covington, J., Egaas, B., Harwood, M., Hinds, S., Press, G.A., 2000. Normal brain development and aging: quantitative analysis at in vivo MR imaging in healthy volunteers1. Radiology 216, 672-682.

Crescentini, C., Shallice, T., Del Missier, F., Macaluso, E., 2010. Neural correlates of episodic retrieval: an fMRI study of the part-list cueing effect. Neuroimage 50, 678-692.

Dale, A.M., Fischl, B., Sereno, M.I., 1999. Cortical surface-based analysis. I. Segmentation and surface reconstruction. Neurolmage 9, 179-194.

Davatzikos, C., Fan, Y., Wu, X., Shen, D., Resnick, S.M., 2008. Detection of prodromal Alzheimer's disease via pattern classification of magnetic resonance imaging. Neurobiol. Aging 29, 514-523.

Delis, D., Kramer, J., Kaplan, E., Ober, B., 1987. California Verbal Learning Test. Psychological Corporation Harcourt Brace Jovanovich, San Antonio.

Desikan, R.S., Segonne, F., Fischl, B., Quinn, B.T., Dickerson, B.C., Blacker, D., Buckner, R.L., Dale, A.M., Maguire, R.P., Hyman, B.T., Albert, M.S., Killiany, R.J., 2006. An automated labeling system for subdividing the human cerebral cortex on MRI scans into gyral based regions of interest. Neuroimage 31, 968-980.
Drane, D.L., Yuspeh, R.L., Huthwaite, J.S., Klingler, L.K., 2002. Demographic characteristics and normative observations for derived-trail making test indices. Neuropsychiatry Neuropsychol. Behav. Neurol. 15, 39-43.

Duarte, A., Hayasaka, S., Du, A., Schuff, N., Jahng, G.-H., Kramer, J., Miller, B., Weiner, M. 2006. Volumetric correlates of memory and executive function in normal elderly, mild cognitive impairment and Alzheimer's disease. Neurosci. Lett. 406, 60-65.

Dwyer, D.M., Dunn, M.J., Rhodes, S.E.V., Killcross, A.S., 2009. Lesions of the prelimbic prefrontal cortex prevent response conflict produced by action-outcome associations. Q. J. Exp. Psychol. 63, 417-424.

Elderkin-Thompson, V., Ballmaier, M., Hellemann, G., Pham, D., Kumar, A., 2008 Executive function and MRI prefrontal volumes among healthy older adults. Neuropsychology 22, 626-637.

Eslinger, P.J., Flaherty-Craig, C.V., Benton, A.L., 2004. Developmental outcomes after early prefrontal cortex damage. Brain Cogn. 55, 84-103.

Fischl, B., Sereno, M.I., Dale, A.M., 1999. Cortical surface-based analysis. II: Inflation, flattening, and a surface-based coordinate system. Neuroimage 9, 195-207.

Fischl, B., van der Kouwe, A., Destrieux, C., Halgren, E., Segonne, F., Salat, D.H., Busa, E., Seidman, L.J., Goldstein, J., Kennedy, D., Caviness, V., Makris, N., Rosen, B., Dale, A.M., 2004. Automatically parcellating the human cerebral cortex. Cereb. Cortex 14, $11-22$.

Foster, J.K., Meikle, A., Goodson, G., Mayes, A.R., Howard, M., Sunram, S.I., Cezayirli, E., Roberts, N., 1999. The hippocampus and delayed recall: bigger is not necessarily better? Memory 7, 715-732.

Fox, N.C., Cousens, S., Scahill, R., Harvey, R.J., Rossor, M.N., 2000. Using serial registered brain magnetic resonance imaging to measure disease progression in Alzheimer Disease: power calculations and estimates of sample size to detect treatment effects. Arch. Neurol. 57, 339-344.

Freeman, S.H., Kandel, R., Cruz, L., Rozkalne, A., Newell, K., Frosch, M.P., Hedley-Whyte E.T., Locascio, J.J., Lipsitz, L.A., Hyman, B.T., 2008. Preservation of neuronal number despite age-related cortical brain atrophy in elderly subjects without Alzheimer disease. J. Neuropathol. Exp. Neurol. 67, 1205-1212.

Ge, Y., Grossman, R.I., Babb, J.S., Rabin, M.L., Mannon, L.J., Kolson, D.L., 2002. Age-related total gray matter and white matter changes in normal adult brain. Part I: volumetric MR imaging analysis. AJNR Am. J. Neuroradiol. 23, 1327-1333.

Gogtay, N., Giedd, J.N., Lusk, L., Hayashi, K.M., Greenstein, D., Vaituzis, A.C., Nugent, T.F., Herman, D.H., Clasen, L.S., Toga, A.W., Rapoport, J.L., Thompson, P.M., 2004 Dynamic mapping of human cortical development during childhood through early adulthood. Proc. Natl Acad. Sci. USA 101, 8174-8179.

Good, C.D., Johnsrude, I.S., Ashburner, J., Henson, R.N.A., Friston, K.J., Frackowiak, R.S.J., 2001. A voxel-based morphometric study of ageing in 465 normal adult human brains. Neuroimage 14, 21-36.

Gunning-Dixon, F.M., Raz, N., 2003. Neuroanatomical correlates of selected executive functions in middle-aged and older adults: a prospective MRI study. Neuropsychologia 41, 1929-1941.

Han, X., Jovicich, J., Salat, D., van der Kouwe, A., Quinn, B., Czanner, S., Busa, E., Pacheco, J., Albert, M., Killiany, R., Maguire, P., Rosas, D., Makris, N., Dale, A., Dickerson, B. Fischl, B., 2006. Reliability of MRI-derived measurements of human cerebral cortical thickness: the effects of field strength, scanner upgrade and manufacturer. Neuroimage 32, 180-194.

Haug, H., Eggers, R., 1991. Morphometry of the human cortex cerebri and corpus striatum during aging [comment] Neurobiol. Aging 12, 336-338 (discussion 352-335).

Head, D., Rodrigue, K.M., Kennedy, K.M., Raz, N., 2008. Neuroanatomical and cognitive mediators of age-related differences in episodic memory. Neuropsychology 22, 491-507.

Head, D., Kennedy, K.M., Rodrigue, K.M., Raz, N., 2009. Age differences in perseveration: cognitive and neuroanatomical mediators of performance on the Wisconsin Card Sorting Test. Neuropsychologia 47, 1200-1203.

Hinton-Bayre, A., Geffen, G., 2005. Comparability, reliability, and practice effects on alternate forms of the Digit Symbol Substitution and Symbol Digit Modalities tests. Psychol. Assess. 17, 237-241.

Hofer, S.M., Sliwinski, M.J., 2001. Understanding ageing. Gerontology 47, 341-352.

Iverson, G.L., 2001. Interpreting change on the WAIS-III/WMS-III in clinical samples. Arch. Clin. Neuropsychol. 16, 183-191.

Jorm, A.F., Anstey, K.J., Christensen, H., Rodgers, B., 2004. Gender differences in cognitive abilities: the mediating role of health state and health habits. Intelligence 32, 7-23.

Köhler, S., Black, S.E., Sinden, M., Szekely, C., Kidron, D., Parker, J.L., Foster, J.K. Moscovitch, M., Wincour, G., Szalai, J.P., Bronskill, M.J., 1998. Memory impairments associated with hippocampal versus parahippocampal-gyrus atrophy: an MR volumetry study in Alzheimer's disease. Neuropsychologia 36, 901-914.

MacKinnon, D.P., Krull, J.L., Lockwood, C.M., 2000. Equivalence of the mediation, confounding and suppression effect. Prev. Sci. 1, 173-181.

MacLullich, A.M.J., Ferguson, K.J., Deary, I.J., Seckl, J.R., Starr, J.M., Wardlaw, J.M., 2002 Intracranial capacity and brain volumes are associated with cognition in healthy elderly men [see comment] Neurology 59, 169-174.

Marner, L., Nyengaard, J.R., Tang, Y., Pakkenberg, B., 2003. Marked loss of myelinated nerve fibers in the human brain with age. J. Comp. Neurol. 462, 144-152.

Mather, K., Jorm, A., Anstey, K., Milburn, P., Easteal, S., Christensen, H., 2010. Cognitive performance and leukocyte telomere length in two narrow age-range cohorts: a population study. BMC Geriatr. 10, 62.

Miller, E.K., Cohen, J.D., 2001. An integrative theory of prefrontal function. Annu. Rev. Neurosci. 24, 167-202.

Müller, N.G., Knight, R.T., 2006. The functional neuroanatomy of working memory: contributions of human brain lesion studies. Neuroscience 139, 51-58.

Oosterman, J.M., Vogels, R.L.C., van Harten, B., Gouw, A.A., Poggesi, A., Scheltens, P. Kessels, R.P.C., Scherder, E.J.A., 2010. Assessing mental flexibility: neuroanatomical 
and neuropsychological correlates of the Trail Making Test in elderly people. Clin. Neuropsychol. 24, 203-219.

Paolo, A.M., Troster, A.I., Ryan, J.J., 1997. Test-retest stability of the California Verbal Learning Test in older persons. Neuropsychology 11, 613-616.

Picton, T.W., Stuss, D.T., Alexander, M.P., Shallice, T., Binns, M.A., Gillingham, S., 2007. Effects of focal frontal lesions on response inhibition. Cereb. Cortex 17, 826-838.

Raz, N., Gunning-Dixon, F.M., Head, D., Dupuis, J.H., Acker, J.D., 1998. Neuroanatomical correlates of cognitive aging: evidence from structural magnetic resonance imaging. Neuropsychology 12, 95-114.

Raz, N., Briggs, S.D., Marks, W., Acker, J.D., 1999. Age-related deficits in generation and manipulation of mental images: II. The role of dorsolateral prefrontal cortex. Psychol. Aging 14, 436-444.

Raz, N., Lindenberger, U., Rodrigue, K.M., Kennedy, K.M., Head, D., Williamson, A., Dahle, C., Gerstorf, D., Acker, J.D., 2005. Regional brain changes in aging healthy adults: general trends, individual differences and modifiers. Cereb. Cortex 15, 1676-1689.

Raz, N., Lindenberger, U., Ghisletta, P., Rodrigue, K.M., Kennedy, K.M., Acker, J.D., 2008. Neuroanatomical correlates of fluid intelligence in healthy adults and persons with vascular risk factors. Cereb. Cortex 18, 718-726.

Resnick, S.M., Pham, D.L., Kraut, M.A., Zonderman, A.B., Davatzikos, C., 2003. Longitudinal magnetic resonance imaging studies of older adults: a shrinking brain. J. Neurosci. 23, 3295-3301.

Riddle, W.R., DonLevy, S.C., Lee, H., 2010. Modeling brain tissue volumes over the lifespan: quantitative analysis of postmortem weights and in vivo MR images. Magn. Reson. Imaging 28, 716-720.

Rodrigue, K.M., Raz, N., 2004. Shrinkage of the entorhinal cortex over five years predicts memory performance in healthy adults. J. Neurosci. 24, 956-963.

Rossi, R., Geroldi, C., Bresciani, L., Testa, C., Binetti, G., Zanetti, O., Frisoni, G.B., 2007. Clinical and neuropsychological features associated with structural imaging patterns in patients with mild cognitive impairment. Dement. Geriatr. Cogn. Disord. 23, 175-183.

Salat, D.H., Kaye, J.A., Janowsky, J.S., 2002. Greater orbital prefrontal volume selectively predicts worse working memory performance in older adults. Cereb. Cortex 12, 494-505.

Salthouse, T.A., 2003. Memory aging from 18 to 80. Alzheimer Dis. Assoc. Disord. 17, $162-167$.

Sanfilipo, M., Lafargue, T., Rusinek, H., Arena, L., Loneragan, C., Lautin, A., Rotrosen, J. Wolkin, A., 2002. Cognitive performance in schizophrenia: relationship to regional brain volumes and psychiatric symptoms. Psychiatry Res. Neuroimaging 116, 1-23.

Schretlen, D., Pearlson, G.D., Anthony, J.C., Aylward, E.H., Augustine, A.M., Davis, A Barta, P., 2000. Elucidating the contributions of processing speed, executive ability, and frontal lobe volume to normal age-related differences in fluid intelligence. J. Int. Neuropsychol. Soc. 6, 52-61.

Shenkin, S.D., Bastin, M.E., MacGillivray, T.J., Deary, I.J., Starr, J.M., Wardlaw, J.M., 2003. Childhood and current cognitive function in healthy 80 -year-olds: a DT-MRI study. NeuroReport 14, 345-349.

Sliwinski, M., Buschke, H., 1999. Cross-sectional and longitudinal relationships among age, cognition, and processing speed. Psychol. Aging 14, 18-33.
Smith, A., 1982. Symbol Digit Modalities Test (SDM) manual. Western Psychological Sciences, Los Angeles.

Soderlund, H., Nyberg, L., Nilsson, L.G., 2004. Cerebral atrophy as predictor of cognitive function in old, community-dwelling individuals. Acta Neurol. Scand. 109, 398-406.

Solé-Padullés, C., Bartrés-Faz, D., Junqué, C., Vendrell, P., Rami, L., Clemente, I.C., Bosch, B., Villar, A., Bargalló, N., Jurado, M.A., Barrios, M., Molinuevo, J.L., 2009. Brain structure and function related to cognitive reserve variables in normal aging, mild cognitive impairment and Alzheimer's disease. Neurobiol. Aging 30, 1114-1124.

Sowell, E.R., Delis, D., Stiles, J., Jernigan, T.L., 2001. Improved memory functioning and frontal lobe maturation between childhood and adolescence: a structural MRI study. J. Int. Neuropsychol. Soc. 7, 312-322.

Staff, R.T., Murray, A.D., Deary, I.J., Whalley, L.J., 2004. What provides cerebral reserve? Brain 127, 1191-1199.

Staff, R.T., Murray, A.D., Deary, I.J., Whalley, L.J., 2006. Generality and specificity in cognitive aging: a volumetric brain analysis. Neuroimage 30, 1433-1440.

Stern, Y., Habeck, C., Moeller, J., Scarmeas, N., Anderson, K.E., Hilton, H.J., Flynn, J., Sackeim, H., van Heertum, R., 2005. Brain networks associated with cognitive reserve in healthy young and old adults. Cereb. Cortex 15, 394-402.

Tisserand, D., Van Boxtel, M., Gronenschild, E., Jolles, J., 2001. Age-related volume reductions of prefrontal regions in healthy individuals are differential. Brain Cogn. 47, 182-185.

Tisserand, D.J., van Boxtel, M.P.J., Pruessner, J.C., Hofman, P., Evans, A.C., Jolles, J., 2004. A voxel-based morphometric study to determine individual differences in gray matter density associated with age and cognitive change over time. Cereb. Cortex 14, 966-973.

Van Der Flier, W.M., Van Den Heuvel, D.M.J., Weverling-Rijnsburger, A.W.E., Spilt, A., Bollen, E.L.E.M., Westendorp, R.G.J., Middelkoop, H.A.M., Van Buchem, M.A., 2002. Cognitive decline in $\mathrm{AD}$ and mild cognitive impairment is associated with global brain damage. Neurology 59, 874-879.

Van Petten, C., 2004. Relationship between hippocampal volume and memory ability in healthy individuals across the lifespan: review and meta-analysis. Neuropsychologia 42, 1394-1413.

Van Petten, C., Plante, E., Davidson, P.S.R., Kuo, T.Y., Bajuscak, L., Glisky, E.L., 2004. Memory and executive function in older adults: relationships with temporal and prefrontal gray matter volumes and white matter hyperintensities. Neuropsychologia 42, 1313-1335.

Wechsler, D., 1945. A standardized memory scale for clinical use. J. Psychol. 19, 87-95.

West, R.L., 1996. An application of prefrontal cortex function theory to cognitive aging. Psychol. Bull. 120, 272-292.

Wimber, M., Bauml, K.-H., Bergstrom, Z., Markopoulos, G., Heinze, H.-J., RichardsonKlavehn, A., 2008. Neural markers of inhibition in human memory retrieval. J. Neurosci. 28, 13419-13427.

Zimmerman, M.E., Brickman, A.M., Paul, R.H., Grieve, S.M., Tate, D.F., Gunstad, J., Cohen, R.A., Aloia, M.S., Williams, L.M., Clark, C.R., Whitford, T.J., Gordon, E., 2006. The relationship between frontal gray matter volume and cognition varies across the healthy adult lifespan. Am. J. Geriatr. Psychiatry 14, 823-833. 